\title{
HIGH RESOLUTION CORONOGRAPHIC IMAGING OF AG CARINAE ${ }^{1}$
}

\author{
ANTONELLA NOTA ${ }^{2}$ and FRANCESCO PARESCE ${ }^{3}$ \\ Space Telescope Science Institute, \\ 3700 S.Martin Drive, \\ Baltimore, $M D 21218$.
}

\begin{abstract}
We present the first high resolution observations of the ring nebula around the galactic LBV AG Car obtained through broad band B, V, R, I filters with the STScI coronograph on the ESO $2.2 \mathrm{~m}$. telescope. Highly anisotropic continuum emission in all these bands is clearly detected, in the shape of a bipolar structure elongated along the NESW direction extending from a few arcsecs from the star out to $\simeq 15$ arcsecs corresponding to a projected linear distance of $\simeq 0.5$ parsecs, with a surface brightness in the range 17.9 $19.4 \mathrm{mag} / \operatorname{arcsec}^{2}$. Its essentially neutral color with respect to the $\operatorname{star}(\Delta(\mathrm{V}-\mathrm{I})=-0.07)$ suggests that the observed radiation is starlight scattered by dust grains of diameter $>1 \mu$.
\end{abstract}

\section{Introduction}

The shell of line emitting gas surrounding AG Car was first detected by Thackeray in 1950 , appearing as an elliptical ring of $39^{\prime \prime} \times 30^{\prime \prime}$ size, with major axis in $\mathrm{PA} \simeq 150^{\circ}$. Its first spectroscopic observations (Johnson, 1976) showed an almost complete absence of [OIII], as might be expected for an evolved object, and the presence of strong $\mathrm{H} \alpha$ to $\mathrm{H} \delta,[\mathrm{NII}] 6548,6584$. Subsequent spectroscopic studies (Thackeray, 1977) derived a remarkable splitting of the $[\mathrm{NII}]$ lines $(\Delta \mathrm{v} \simeq 119 \mathrm{~km} / \mathrm{sec})$ occurring not along the radial expansion direction but parallel to the shell indicating that some portions of the nebula were site of violent motions concentrated close to the star and in a portion of the ring, probably due to a mass loss effect still persisting after the outburst that created the nebula. Recent CCD observations by Stahl (1987) in the light of the nebular emission lines $\mathrm{H} \alpha$ and [NII] have shown the asymmetry of the shell, but without reaching the resolution and the contrast necessary to resolve the structures in the inner part of the ring, due to the brightness of the central star. The problem is even more dramatic in the continuum, where the light of the star $\left(m_{V} \simeq 7.7\right)$ dominates the nebula even in a few seconds exposure.

A detailed investigation of the AG Car nebula in the light of the continuum is fundamental, however, to reveal the presence of other emission mechanisms such as

\footnotetext{
${ }^{1}$ Based on data collected at the European Southern Observatory, La Silla, Chile.

${ }^{2}$ On leave from the Observatory of Padova, Italy.

${ }^{3}$ Affiliated to the Astrophysics Division, Space Science Department, ESA
}

\section{9}

K. Davidson et al. (eds.), Physics of Luminous Blue Variables, 159-164.

(C) 1989 by Kluwer Academic Publishers. 
non-thermal or dust-scattered radiation. IR and UV data have recently confirmed that there is a substantial amount of dust in the shell. Observations in the far infrared (McGregor et al., 1988), show evidence of two well resolved peaks at 50 and $100 \mu$, located at about 9 arcsecs from the star, respectively in direction SW and NE and interpreted by the those authors as being due to a clumpy distribution of cool graphite and silicate dust grains of size $>1 \mu$. IUE observations of the nebula (Viotti, 1987) show a nearly constant nebular/stellar continuum ratio between 2000 and $3000 \AA$ suggesting that the main source of UV radiation is the scattering of stellar light by dust particles in the nebula.

\section{Observations and Data Reduction}

Accurate observations of the two dimensional dust distribution around an object this bright $\left(\mathrm{m}_{V} \simeq 7.7\right)$ down to a few arcsecs from the star are hampered by the glare from the central star that swamps the faint nebular signal in a broad enough band to detect the scattered continuum. This problem has been recently overcome by using the STScI coronograph mounted on the ESO/MPI $2.2 \mathrm{~m}$. telescope at La Silla.

Briefly, the observing configuration of the coronograph is the following: the light of the central star is focussed onto an occulting mask located in the telescope focal plane (Paresce, Burrows and Horne, 1987). This mask is shaped in the form of a long, thin wedge, that can be moved longitudinally by a micrometer in order to vary its projected width on the sky from 1 to 10 arcsecs depending on the seeing conditions and source brightness. An achromatic doublet reimages the telescope focal plane with a magnification of 5 onto the detector so that the effective focal ratio of the system becomes $\mathrm{f} / 40$. A specially designed apodizing mask reduces the stellar light diffracted from the $2.2 \mathrm{~m}$ telescope pupil. The detector used is a RCA CCD of $320 \times 51230$ micron pixels, giving an unrestricted field of view of $22.5 \times 36$ arcsecs, with a pixel size of $7.03 \times 10^{-2}$ arcsecs.

AG Car was observed on 1987 December 21 in this configuration, in the broad band pass filters $B, V, R, I$ and in the narrow band interference filters centered on the $H \alpha$, at $6563 \AA,[\mathrm{NII}]$ at $6583 \AA$, and [SII] at $6716 \AA$. The wavelength dependance of the response of these filters closely resembles those of the standard Johnson $B, R$ and the Cousins $I_{c}$ filters. The complete system has been calibrated absolutely using a standard stellar calibration source (Feige 25) observed in the same broad band filters. The V filter $\left(\lambda_{o}=5591.5, \Delta \lambda=744 \AA\right)$ was chosen such that its bandpass is in a region free of emission lines in order to avoid contamination from line emission: comparison calibrations on Feige 25 with the standard Johnson V have shown that our narrow $V$ magnitude is for all purpose identical to the standard $V$ magnitude.

AG Car is first imaged in the unocculted portion of the field and with a neutral density filter of optical density 2.0 and the narrow $V$ filter in order to provide an estimate of its flux at observation time $\left(m_{V}=7.7\right.$, consistent with the AAVSO measurements for that epoch). Then, using the telescope TV autoguiding system, AG Car is placed behind the occulting wedge in a series of short acquisition exposures. The centering of the star behind the mask is achieved by commanding increasingly smaller offsets to the telescope till the spillover light distribution above and below 
the wedge becomes as symmetrical as possible; at this point, all the neutral density filters are removed and the scientific exposures started. Due to the excellent seeing conditions (FWHM in focus $\simeq 0.77$ arcsecs) the occulting wedge was set to a width at the center of the stellar profile of $\simeq 2$ arcsecs, running in the direction EW.

All the images were preprocessed according to standard procedures. Bad columns, blemishes and cosmic rays have been removed from all the frames, then the CCD bias level has been measured in the overscan columns of the frames, averaged and removed. The frames have then been dark subtracted and flat fielded. Results of the processing are shown in Fig.1-a,b. The image on the left corresponds to a $10 \mathrm{~min}$. exposure in the narrow $\mathrm{V}$ band and on the right to a $10 \mathrm{~min}$. exposure in the $\mathrm{H} \alpha$ band; north is up, east is to the left, and the cross marks the geometric center of the stellar image. No further image processing has been carried out on these images: diffraction spikes are still visible in the NW-SE, $N, N E$ directions from the star. In all the frames the occulting wedge has been masked out in order to avoid software problems in correspondance to the saturated pixels at its edges.

\section{Analysis and Discussion}

As we can see from Fig.1a, the images taken in the broad band continua provide a completely different view of the AG Car system, with respect to the well defined ring-shaped nebula observed in the light of the nebular emission lines (Fig.1b). In the continuum there is unambiguous evidence of a jet-like structure extending in direction SW from a distance of approximately 5 arcsecs from the center of the star towards the ring at $\simeq 15$ arcsecs from the center. This feature is extremely well defined in the B, V, I images and seems to consist of two thick helical filaments spiralling towards the external portion of the nebula. It is not visible at all in any of the narrow band images, its shape and size are apparently wavelength independent, and its intensity remains approximately constant with increasing distances from the center. In the opposite direction, towards the $\mathrm{NE}$, at a distance of approximately 9.5 arcsecs from the central star, there is evidence of an extended feature of comparable surface brightness, completely detached from the star and immersed in the weak ring nebulosity. Its sharp inner boundary is reminiscent of the interaction region between a hot stellar wind and the remnants of previous ejecta. The characteristic shell nebula, bright and well defined in the nebular line images, is barely visible, and shows abrupt shock-like edges in the NE and NW inner regions. At the center of the image, the glow due to the scattered light distribution of the central star, not totally suppressed by the coronograph, is still evident, extending to $\simeq 4$ arcsecs from the center of the star. An investigation of the behaviour of the dust distribution in the closest portion to the star has been attempted by subtracting from the $\mathrm{V}$ frame a suitably chosen and scaled reference star, in order to suppress the central glow. Irregularities in the PSF do not allow us to understand if the dust extends closer than 5 arcsecs from the star. However, it is clear that more material is concentrated in the SW direction, and much closer to the star with respect to the NE feature.

The narrow band image in $\mathrm{H} \alpha$ (Fig.1b) shows the nebular structure at unprecedented resolution. The two known areas of highest surface brigthness are well visible 
along the SW-NE axis. In addition, it seems possible to identify, especially in the NW and SW regions, an inner ring partially detached from the outer, thicker shell, suggestive of two different, subsequent outbursts experienced by the star in its mass loss history. The same details are found in the [NII] image, even if at a lower sig$\mathrm{nal} /$ noise. A comparison with the broad band images indicates that the dusty region of the jet-structure roughly overlaps with the brightest region of the emission nebula; however, there is no evidence of an helical structure in the nebular lines. A comparison with the data published by McGregor et al., within the limits allowed by the resolution of the infrared measurements, indicates that the brighter $\mathrm{SW}$ peak concides with the brightest area of the jet, at $\simeq 9-10$ arcsecs from the star, the NE peak is approximately located in the region of the counterjet.

An estimate of the integrated flux of the jet structure gives $\mathrm{m}_{V}=17.94 \mathrm{mag} / \mathrm{arcsec}^{2}$ and $\mathrm{m}_{I}=17.25 \mathrm{mag} / \mathrm{arcsec}^{2}$, in the brightest area. Assuming the photometry published by McGregor et al. (1985), [(V-I) star $=0.76]$ we obtain: $(\mathrm{V}-\mathrm{I})_{j e t}=0.69, \Delta(\mathrm{V}-\mathrm{I})$ $=-0.07$, where $\Delta$ is the colour difference between the jet and the star. Within the uncertainties, these values seem compatible with a stellar light scattering model due to large dust particles distributed between the star and the nebula and not yet swept up by the stellar wind.

\section{Acknowledgements}

We wish to thank all the people who have contributed to the realization of this project: C.Burrows, D.Hofstadt and the ESO operations group at La Silla; A.Ferrari, D.Shore, K.Davidson for useful discussions. We acknowledge the AAVSO observational data sent to us by the Director of the AAVSO, Janet A. Mattei. We are particularly grateful to Roberto Viotti for suggesting the observation of AG Car.

\section{References}

Johnson, H. M. (1976) 'Spectra and Fabry-Perot interferometry of AG Car and the nebula', Astrophys. J., 206, 469.

McGregor, P.J., Finlayson, K., Hyland, A.R., Joy M., Harvey, P. M., Lester, D.F. (1988) 'Far infrared emission from the AG Carinae ring', Astrophys. J. 329, 874.

Paresce, F., Burrows, C., Horne, K. (1988) 'The structure of the inner R Aquarii nebula in the light of $\mathrm{H} \alpha$ and [NII] $\lambda 6584 \AA$ emission', Astrophys. J., 329, 318.

Stahl, O. (1987) 'Direct imagery of circumstellar shells around Ofpe/WN9 stars in the galaxy and in the LMC', Astron. Astrophys., 182, 229.

Thackeray, A.D. (1950) 'Some southern stars involved in nebulosity', Monthly Notices Roy. Astron. Soc., 110, 524.

Thackeray, A.D. (1977) 'Spectra of the low-excitation nebulosities around AG Carinae and HD 138403', Monthly Notices Roy. Astron. Soc., 180, 95.

Viotti, R., Cassatella, A., Ponz,D., The, P.S. (1987) 'AG Carinae. II. IUE observations of the nebula', Astron. Astrophys., 190, 333. 


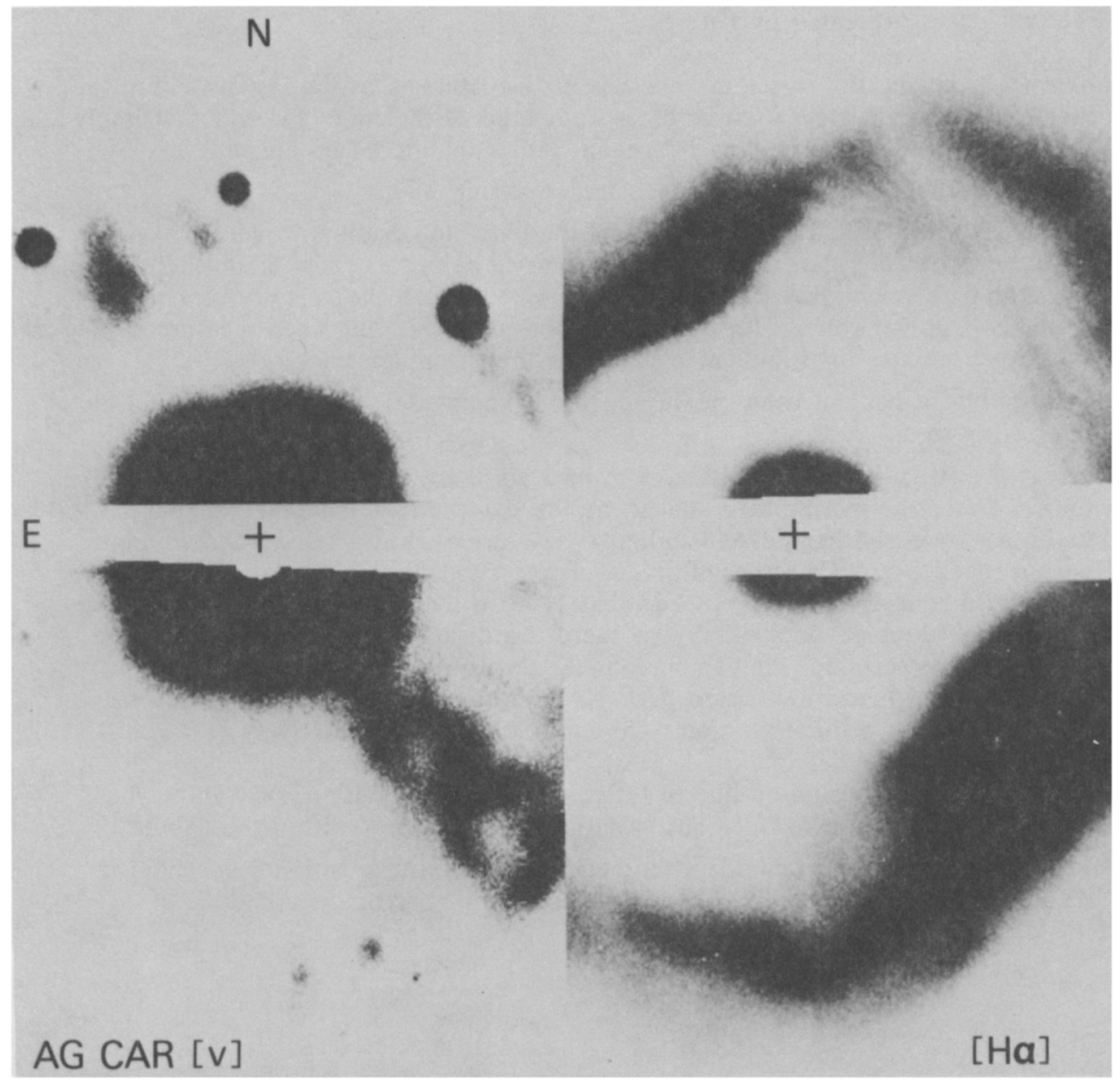

Fig.1a,b - on the left, 10 min. exposure in the V band of the AG Car system. A cross marks the center of the star, whose light is almost completely suppressed by the coronograph. The occulting wedge has a width at the center of $\simeq 2$ arcsecs. A jet-like structure is well visible in direction SW from a distance of approximately 5 arcsecs from the star towards the outer portion of the ring. On the right, $10 \mathrm{~min}$. image of the characteristic shell nebula in the light of $\mathrm{H} \alpha$. 


\section{DISCUSSION}

[The paper was presented by Paresce.]

Lamers: It is amazing to see in your beautiful pictures that the region within the ring in [N II]-light is so faint or black compared to the ring. Doesn't that imply that we are seeing a ring almost face-on, rather than a spherical shell?

Paresce: Perhaps, but if so, a very asymmetric ring.

Owocki: It seems remarkable that one of the most outstanding objects in the sky should eject a ring of material perpendicular to our line of sight. If this is true then the true velocity of ring expansion is greater than the velocity measured from the Doppler shift. Has the proper motion of this ring been measured? Is it much greater than implied by the measured Doppler velocity?

Paresce: No, it has not been measured, to my knowledge. It certainly would be interesting to do so.

Davidson: It doesn't absolutely have to be a ring, not with those jets pointed like that. To me, one morphology evoked by the two pictures together is like a pair of umbrellas, opposed handle-to-handle, $\leftarrow \rightarrow$, oriented at a fairly random angle with respect to us. Or a pair of jets squirting fluid toward opposite points on the inside of a spherical fishbowl. And that helix in the SW jet is marvelous -- why does it look double? Incidentally, if there is a disk and a jet and a companion star causing precession, the companion star's orbit period will be the geometric mean of the disk and precession periods, in an order-of-magnitude sense: maybe $\sim 20$ years or so, with separation $\sim 20$ a.u. or so, very speculatively!

Heap: (1) Is the continuum due to reflection or recombination? (2) Have you used the $\mathrm{H} \alpha / \mathrm{H} \beta$ ratio to trace out variations of extinction within the nebula?

Paresce: All of the available data indicate reflection as the emission mechanism. We have not taken the $\mathrm{H} \alpha / \mathrm{H} \beta$ ratio as you suggest, but this is a good idea.

Francesco Paresce

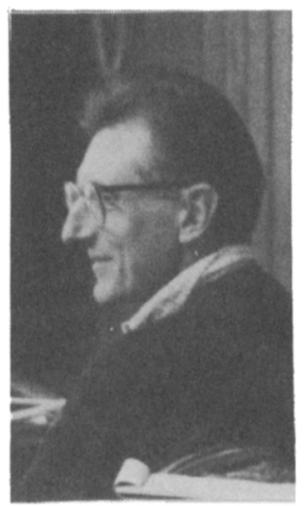

